\title{
On favorable thermal fields for detached Bridgman growth
}

\author{
Carmen Stelian ${ }^{a}$, Martin P. Volz ${ }^{b}$, and Jeffrey J. Derby ${ }^{c, *}$ \\ ${ }^{a}$ Department of Physics, West University of Timisoara, Bd. V. Parvan, No. 4, 300223 Timisoara, Romania \\ ${ }^{b}$ NASA Marshall Space Flight Center, EM30, Huntsville, AL 35812, USA \\ ${ }^{c}$ Department of Chemical Engineering 83 Materials Science, University of Minnesota, \\ Minneapolis, MN 55455-0132, USA
}

\begin{abstract}
The thermal fields of two Bridgman-like configurations, representative of real systems used in prior experiments for the detached growth of CdTe and Ge crystals, are studied. These detailed heat transfer computations are performed using the CrysMAS code and expand upon our previous analyses [14] that posited a new mechanism involving the thermal field and meniscus position to explain stable conditions for dewetted Bridgman growth. Computational results indicate that heat transfer conditions that led to successful detached growth in both of these systems are in accordance with our prior assertion, namely that the prevention of crystal reattachment to the crucible wall requires the avoidance of any undercooling of the melt meniscus during the growth run. Significantly, relatively simple process modifications that promote favorable thermal conditions for detached growth may overcome detrimental factors associated with meniscus shape and crucible wetting. Thus, these ideas may be important to advance the practice of detached growth for many materials.
\end{abstract}

PACS: 07.05.Tp; 81.10.-h; 81.10.Fq

Keywords: A1. Computer simulation; A1. Heat transfer; A2. Bridgman technique; A2. Growth from melt

${ }^{*}$ Corresponding author. Fax: +1-612-626-7246; e-mail: derby@umn.edu 


\section{Introduction}

The detached Bridgman growth is a new technique of crystal growth without crucible contact. The dewetting phenomenon was observed for the first time in microgravity experiments $[1,2]$ then successfully reproduced in earth experiments [3-10]. The prevailing wisdom for conducting detached growth on earth is to establish a liquid meniscus between the crystal and the crucible wall and maintain it by controlling the pressure difference between the gas space above the melt and the gap. This practice follows early theoretical analyses of the stability of the process that concentrated on meniscus mechanics and indicated that dewetted growth depends on the wetting angles of the melt with the ampoule and the crystal and the pressure applied along the gas-liquid meniscus [11-13].

However, many of the experiments conducted at favorable theoretical growth conditions according to meniscus mechanics have shown reattachment events during the growth process. In a prior thermal-capillary analysis of detached Bridgman growth [14], we have put forth a new explanation for process stability that invokes heat transfer arguments to explain reattachment events that would end dewetting. According to our prior results, crystal reattachment to the crucible wall is explained by the freezing of the liquid meniscus between the growing crystal and the crucible wall, which occurs when the solidification isotherm passes above the liquid-gas surface of the meniscus. This work also identified possible remedies to prevent freezing of the meniscus, including changing the meniscus shape via wetting angle changes and increasing the pressure under the meniscus (although this approach is limited by the possibility of meniscus rupture by gas bubbling). A more robust approach is the tailoring of thermal conditions to coax the solidification isotherm to pass through the gap, under the meniscus, rather than through the melt, above the meniscus. Such conditions were shown in [14] to correspond with the promotion of convex solid-liquid interface shapes.

The present work is meant to extend the understanding provided from our prior analysis [14] by focussing on thermal conditions associated with furnace design. We carry out detailed heat transfer computations for two configurations like those employed in prior experimental studies of the detached growth of germanium by Palosz et al. [9] and cadmium telluride by Chevalier et al. [5]. Our analyses reinforce the notion that favorable thermal conditions may prevent reattachment during detached growth.

\section{Model}

Our prior analysis employed a rigorous, thermal-capillary model that concentrated on the detailed coupling of interfacial and field phenomena but applied a very simplified representation of furnace heat transfer [14]. Here, we apply the sophisticated CrysMAS code developed by the Crystal Growth Laboratory of the Fraunhofer Institute of Integrated Systems and Device Technology (IISB) in Erlangen, Germany [15] to perform rigorous simulation of heat transfer in multi-zone Bridgman furnaces. While this promises a much greater degree of realism with respect to modeling furnace heat transfer, the drawback of this approach is that we do not fully represent the coupling of meniscus dynamics with the system. Rather, here we simply prescribe the position and shape of the meniscus based on our prior results [14]; more details are provided below. Neither our prior modeling approach [14] nor the one taken here is entirely correct; however, both reveal interesting behavior and useful understanding of these complex systems.

We apply CrysMAS for the global modeling of the heat transfer in the whole system, including the ampoule and contents, the furnace, and thermal shields. We consider heat transfer occurring by conduction and radiation in an axisymmetric configuration. Since our prior analysis indicated 
that convective heat transport in the melt was unimportant in these small-scale systems [14], we choose to ignore melt convection in the current model. We approximate the effects of gas-phase convection in the furnace by employing heat transfer coefficients and effective thermal conductivities in open regions. Radiant heat transfer is computed through all gas-filled regions (including the gaps between the crystal and crucible and between crucible and ampoule) using view factors and enclosure analysis. Unless specifically stated otherwise, radiant transport is assumed to occur through all quartz components and is computed using a semitransparent band approximation, where the material is assumed to be transparent for radiation wavelengths of 1-4 $\mu \mathrm{m}$ and opaque for all other wavelengths. A thermal shield, assumed to have a thermal conductivity $k=20$ $\mathrm{Wm}^{-1} \mathrm{~K}^{-1}$ and an emissivity $\epsilon=0.8$, is considered to surround each furnace in our simulations, and a constant ambient temperature of $T=300 \mathrm{~K}$ is imposed as an outer boundary condition. Additional furnace details will be discussed in the results sections.

We employ the powerful inverse simulation mode of CrysMAS to compute input power to resistive heaters in order to satisfy temperature control set points. We also apply this approach to impose the constraint of the solidification temperature to the trijunction point. This allows for the joining of the prescribed fixed-radius crystal, the assumed meniscus shape, and the computed (and varying) solidification interface in a mathematically and physically consistent manner.

Thus, computations proceed in the following manner. A small gap of constant thickness $0.1 \mathrm{~mm}$ is applied between the crystal and the inner crucible. Then the vertical location of the trijunction, where gas, melt, and crystal meet, is specified. As mentioned above, the meniscus shape is not computed by the code but is set by drawing a curve connecting the trijunction and the crucible wall, so that the wetting condition at the wall is attained. Finally, inverse simulation is applied to compute the power to the heaters in order to reach the solidification temperature at the trijunction, which defines the outer terminus of the solid-liquid interface and, in turn, sets the form of the thermal field of the entire system.

\section{$3 \quad$ Results}

\subsection{Two-heater Bridgman furnace}

Figure 1 shows the simplified geometry used to model the two-heater Bridgman furnace employed for the detached growth of the germanium by Palosz et al. [9]. The geometry is considered to be axisymmetric, with the system centerline drawn on the left side of the schematic. The outer silica ampoule has an inner tube containing the charge. Several sets of experiments were conducted in this configuration using different inner tubes, ostensibly to investigate different wetting conditions on detached growth. The furnace has two heating elements separated by an adiabatic zone. To drive directional solidification, the furnace heaters are translated upward at a constant rate.

Palosz et al. [9] found that successful dewetting over the entire growth run only occurred when a pyrolytic boron nitride (BN) inner tube was employed and attributed this success to the high value of the Ge contact angle on $\mathrm{BN}$ surfaces $\left(\theta \approx 170^{\circ}\right)$. Other crucibles proved less successful in attaining stable, detached growth conditions.

\subsubsection{Effect of meniscus shape}

We first consider the conditions of successful detached growth of Ge in BN crucibles. As shown in Figure 2, the specific geometry consists of a BN inner crucible within a quartz ampoule; the gap between the crucible and ampoule is filled with gaseous argon. The position of the trijunction is set to be exactly in the middle of the adiabatic zone by adjusting heater power according to the 
procedures described in Section 2. We concentrate here and in subsequent computations of this system only on the relative position of the solidification isotherm, which is critical to ascertain conditions that may avoid reattachment events [14].

In the detailed view shown on the lower left-hand side of Figure 2, we consider a meniscus shape representative of the real system, with a wetting angle of $\theta \approx 170^{\circ}$ and a convex-concave shape that is comparable to those computed by the thermal-capillary model in [14]. The thermal field in this case shows that the solidification isotherm is very closely aligned with the liquid-gas surface of the meniscus. Although there is a very small region of undercooled melt near the wall wetting point, most of the meniscus is kept at a temperature above the melting point, and this situation is favorable for dewetted growth, as observed in experiments. (Note: as will be seen in Section 3.1.3, the situation becomes more favorable as growth proceeds.)

As a thought experiment, a second computation was carried out with a different meniscus shape but keeping all other parameters identical to the first computation. Here, a much decreased gap pressure and a smaller wetting angle are considered to produce a concave meniscus shape, as seen in the upper detailed view of Figure 2. In this case, the solidification isotherm is positioned above the meniscus, giving rise to a large undercooled zone in the melt. This is expected to quickly solidify, and the crystal will be attached to the crucible. These results demonstrate the importance of the meniscus geometry, as determined by pressure and contact angle, and are consistent with prior stability ideas based on meniscus mechanics $[8,16-19]$. However, the source of instability in this computation has its origins in heat transfer, as argued in [14].

\subsubsection{Effect of ampoule-zone alignment}

Based on the ideas first put forth in [14], we undertook a series of computations to ascertain thermal conditions in this furnace that would enhance the stability of detached growth, especially for meniscus shapes that are less favorable than that obtained in the highly wettable BN crucible. Interesting results are achieved by simply changing heater settings to push the position of the solidliquid interface upward. Figure 3 shows the predicted outcomes for an interface position raised 3.5 $\mathrm{cm}$ above the adiabatic zone and into the hot zone of the furnace.

Insets on the left-hand side of Figure 3 show the relative positions of the meniscus and solidification isotherm for three different crucible configurations. The upper case represents the BN crucible considered in the prior section. Under the new conditions, the solidification isotherm still tracks closely with the meniscus shape; however, the small undercooled melt region observed in the prior case (see Figure 2) is now gone. The middle case represents an inner quartz crucible held tightly within the outer quartz ampoule; the meniscus is assumed to form a straight line and wets the wall at a smaller angle of $\theta \approx 160^{\circ}$. As is evident from the relative position of the solidification isotherm, this configuration presents a more favorable thermal condition than the $\mathrm{BN}$ case, with the isotherm clearly passing below the meniscus. A yet better situation is presented when the space between the inner crucible and the outer ampoule is filled with gas having a low thermal conductivity, as shown in the bottom inset. Here, even the worst-case scenario of a very low pressure with a concave meniscus shape still produces a thermally stable configuration. These results imply that favorable thermal conditions, here obtained by selected ampoule-zone alignment, may promote conditions to achieve and maintain detachment, even for mechanically stable meniscus shapes that are less conducive to detachment. 


\subsubsection{Stable growth}

Finally, we carry out several sets of time-dependent computations that move the heating zones upward to simulate conditions for growth. The inverse simulation for the initial condition supplies power levels for the heaters, which are subsequently held constant as the heating zones are moved and transient results are computed using forward simulation.

Our first set of computations was performed for the BN crucible configuration described in Section 3.1.1, with an initial condition corresponding to the BN case shown in Figure 3. We do not show the outcome of this series of computations but state that stable thermal conditions were obtained for all stages of growth and that the estimated ratio of the solidification growth rate, $V_{g}$, to the furnace translation rate, $V_{t}$, was $V_{g} / V_{t} \approx 1.5$. This compares very favorably to the observed experimental results [9].

We next performed a series of thought experiments designed to test the assertion of the prior section that favorable thermal conditions may be able to compensate for less favorable wetting characteristics. Namely, we consider the outcome of growth using the ampoule-zone alignment of the prior section with quartz-crucible configurations employed by Palosz et al. [9] which were prone to reattachment events in their experiments.

The first of these thought experiments considers the quartz crucible and ampoule with a gap that was discussed in the previous section. Figure 4(a) shows the initial condition for growth, with the interface positioned at $3.5 \mathrm{~cm}$ above the adiabatic zone, corresponding to a seed length of about $2 \mathrm{~cm}$. In this initial state, the solidification isotherm passes through the gaseous gap, as shown in the detailed view. The thermal conditions become more favorable for detached growth when the furnace is translated up, as is shown in Figure 4(b) and (c). In this system, the solidification rate was slightly greater than the $\mathrm{BN}$ case, with $V_{g} / V_{t} \approx 1.66$.

The second thought experiment is depicted in Figure 5. Here, we add a graphite layer between the inner quartz crucible and the outer quartz ampoule. The major effect of the graphite layer replacing the air gap of the prior case is to move the position of the solidification isotherm upward due to the greater amount of heat transfered through the high-conductivity graphite. Though less favorable than the previous case, this system still exhibits thermal stability against reattachment during the entire growth run. Another effect arising from the graphite-promoted heat transport through the crucible-ampoule assembly is that the growth rate is quite similar to the furnace translation rate in this system, with $V_{g} / V_{t} \approx 1.1$.

\subsection{Three-heater vertical gradient freeze furnace}

We next consider an idealized vertical gradient freeze (VGF) configuration with three heaters, shown in Figure 6 and representative of that used for the detached growth of CdTe by Chevalier et al. [5]. As in the prior model, the geometry is considered to be axisymmetric, and the system centerline is drawn on the left side of the schematic. Directional solidification is obtained by powering down the furnace heaters to achieve a constant rate of temperature reduction at control set points.

Chevalier et al. [5] attained successful detached growth only for an optimized thermal field in the furnace. The CdTe charge, with dimensions $1.4 \times 8 \mathrm{~cm}$, was contained in a silica crucible contained by an outer silica ampoule. The furnace had three heating elements put at a distance of $1 \mathrm{~mm}$ from the outer ampoule. The temperature inside the furnace was measured by using thermocouples placed in the space between the crucible and ampoule. According to Chevalier et al. [5], the optimal set of temperatures measured in the experiment at the beginning of the growth process was $T_{1}=1,163 \mathrm{~K}, T_{2}=1,188 \mathrm{~K}$, and $T_{3}=1,373 \mathrm{~K}$. This set of temperatures has been imposed at three control points (see Figure 6), and the power supplied in the furnaces has 
been computed by using inverse simulation.

\subsubsection{Effect of meniscus shape}

We first compute the thermal fields at the beginning of the solidification process, shown in Figure 7, for the optimal case reported in [5]. The position of the solid-liquid interface, calculated from inverse simulation requiring the thermal field to meet the optimal set-point temperatures given previously, corresponds to a seed length of approximately $3.7 \mathrm{~cm}$, which is in good agreement with the experimental report of a seed of $3.5 \mathrm{~cm}$ [5]. The interface is concave with a small deflection of approximately $0.6 \mathrm{~mm}$. The upper detail shows a case where the meniscus shape is set according to our prior thermal-capillary computations for the growth of CdTe in a quartz crucible [14] corresponding to a wetting angle of $\theta=100^{\circ}$ and an applied pressure difference of $\Delta p=-10$ mbar. Under these conditions, the solidification isotherm passes through the gap, just below the meniscus, therefore thermal conditions are favorable to support dewetting at this initial stage of growth. The lower case shows a computation under the same conditions, where the meniscus shape has been simplified as a straight line. Since there is virtually no change to the thermal conditions, the straight-line meniscus shape is used in ensuing computations for this system.

\subsubsection{Effect of crucible heat transfer}

Knowing that dewetted growth in this system was particularly prone to reattachment events for most furnace thermal settings, we consider another series of thought experiments where we examine how thermal stability conditions may be affected by crucible design. In Figure 8, we consider a later stage of growth, corresponding to $5.9 \mathrm{~cm}$ length of the solid, for several different crucible situations.

Figure 8(a) shows the system considered in the prior section that featured a semitransparent quartz crucible. Even though the initial state shown in Figure 7 was stable, crystal reattachment becomes likely by the time the crystal has grown to the height shown in Figure 8(a), as indicated by the thin undercooled liquid layer along the entire meniscus. This somewhat puzzling result is at odds with the reported successful growth experiment of Chevalier et al. [5]. We therefore seek an explanation by examining the details of crucible heat transfer.

To bracket the possible heat transfer behaviors produced by different crucibles, we first consider the case of a quartz ampoule that is totally transparent to radiation, shown as Figure 8(b). Here, stronger cooling caused by increased heat transfer through the crucible makes the undercooled melt layer significantly expand. This situation is less likely to lead to successful growth than the case of the semitransparent crucible.

Better results are obtained when we consider a semitransparent quartz ampoule whose exterior is coated with a layer of opaque graphite, exhibited in Figure 8(c). In this system, thermal conditions move the solidification isotherm downward, so that it passes through the gap rather than the melt. Finally, Figure 8(d) presents a case where the quartz ampoule is considered to be opaque to thermal radiation. This case also offers a stable thermal configuration for maintaining detached growth, with the solidification isotherm passing beneath the melt meniscus. Both of these cases produce outcomes that are more consistent with the reported successful case of Chevalier et al. [5].

\subsubsection{Stable growth}

The thermal field for the opaque crucible case of the prior section (Figure 8c) is very similar to that of the graphite-coated, semitransparent crucible (Figure 8d). Since the opaque-crucibe case is also far less computationally taxing, we choose to employ it in transient computations for a growth run. 
We compute the course of a growth experiment by reducing the temperature of the control points in time. Figure 9(a) show the initial condition, and Figures 9(b) and (c) show times that correspond to a lowering of temperature of $60 \mathrm{~K}$ and $80 \mathrm{~K}$, respectively. Clearly seen in the detailed views is that the solidification isotherm remains always below the meniscus so that the entire growth run exhibits stable conditions against reattachment.

Interestingly, the behavior of this case is very similar to those of the transient Bridgman simulations (see Section 3.1.2), especially with respect to how the thermal field becomes more favorable as the solidification interface position moves higher within the hottest zone of the furnace. Accompanying this more favorable thermal field for dewetted growth is the evolution of a convex solid-liquid interface shape.

\section{Conclusions}

The global modeling of the thermal field in two vertical Bridgman-like crystal growth configurations has been performed to better understand favorable thermal conditions for successful detached growth of Ge and CdTe crystals. From a crystal growth modeling perspective, the model employed previously in [14] was a first step toward a fully integrated thermal-capillary analysis, as has been implemented for other meniscus-defined growth systems [20-26]. The analysis employed here represents another step toward a model that more faithfully depicts the details of heat transfer in technically realistic furnaces. We believe that the next advance will effectively couple these two models with their respective strengths using algorithms that are currently under development [27-30].

The computations for the specific systems considered here show some interesting results. We simulated the vertical Bridgman growth of Ge by Palosz et al. [9] and found, consistent with their results, that the large wetting angle of germanium on boron nitride surfaces was an important factor to promote conditions for stable, detached growth. Surprisingly, our computations predicted that simply reorienting the ampoule with respect to the furnace zones substantially improved the thermal conditions needed to avoid reattachment. By initiating growth much higher into their hot zone, favorable thermal conditions were predicted for continued detachment, even for systems that did not exhibit large wetting angles. In addition, substantial improvements in heat transfer, that affected the placement of the solidification isotherm with respect to the meniscus and hence the prospects for successful detached growth, were attained by relatively simple changes in crucibleampoule design.

Analysis of the vertical gradient freeze configuration developed by Chevalier et al. [5] for the detached growth of CdTe yielded initially puzzling results. Namely, our computations predicted that, despite an initial condition that would support dewetted growth, continued growth under reported conditions would eventually lead to undercooling of the meniscus. These poor stability characteristics were eliminated when the transparency of the inner crucible was altered, either by a graphite coating or by making it opaque to radiation. Hence, we speculate that the success reported in [5] may have arisen from intentionally applied graphitization of the crucible (although such procedures were not reported) or by some source of surface contamination of the crucible that deposited an opaque coating. Improved thermal conditions were also predicted, for coated or opaque crucibles, when the solid-liquid interface moved higher into the hot zone during growth. Thus, the conditions needed to maintain dewetted growth in this system improved in a manner similar to that in the Bridgman system when the growth interface was placed deeper into the hot zone. For both systems, thermal conditions that promoted a convex solid-liquid interface shape also produced favorable conditions to maintain detached growth. 
Our prior work [14] argued that the long-standing notion of dewetted growth stability being determined solely by meniscus mechanics is incomplete and that thermal effects are also profoundly important. The results presented here strengthen that argument and propose important ideas for advancing the practice of detached growth. Clearly, the properties of the meniscus are very important in these systems. First, the meniscus must be mechanically stable to even allow the detached geometry to be established and maintained. Second, large wetting angles between the melt and crucible wall and large applied pressures in the gap are beneficial, since they move the melt adjacent to the meniscus upward and potentially away from the solidification isotherm [14]. What is new about the understanding obtained here is that the effects of less favorable meniscus shapes, such as those that are set by smaller wetting angles or lower applied pressures, that lead to reattachment under nominal conditions, might be ameliorated by the engineering of favorable heat transfer conditions in the system. Though supported only by computational evidence at this point, we propose that relatively simple modifications to current practice may attain thermal conditions allowing contactless growth during the entire process. Thus, these ideas may be important to advance the practice of detached growth for many materials.

\section{Acknowledgments}

This work has been supported in part by the Minnesota Supercomputing Institute and the Department of Energy, National Nuclear Security Administration, under Award Number DE-FG5206NA27498, the content of which does not necessarily reflect the position or policy of the United States Government, and no official endorsement should be inferred. CS acknowledges support from the Alexander Dubcek Fund, administered by the Office of International Programs of the University of Minnesota. JJD wishes to thank J. Friedrich, T. Jung, and G. Ardelean of the Crystal Growth Laboratory, Fraunhofer IISB, for ongoing collaborations involving CrysMAS.

\section{References}

[1] ] J. T. Yue and F.W. Voltmer, J. Crystal Growth 29 (1975) 329.

[2] A.F. Witt, H.C. Gatos, M. Lichtensteiger, M.C. Lavine, and C.J. Herman, J. Electrochem. Soc. 122 (1975) 276.

[3] T. Duffar, P. Dusserre, F. Picca, S. Lacroix and N. Giacometti, J. Crystal Growth, 211 (2000) 434.

[4] T. Duffar, P. Dusserre, N. Giacometti, J. Crystal Growth, 223 (2001) 69.

[5] N. Chevalier, P. Dusserre, J.P. Garandet and T. Duffar, J. Crystal Growth, 261 (2004) 590.

[6] M. Fiederle, T. Duffar, J. P. Garandet, V. Babentsov, A. Fauler, K. W. Benz, P. Dusserre, V. Corregidor, E. Dieguez, P. Delaye, G. Roosen, V. Chevrier, J.C. Launay, J. Crystal Growth, 267, 2004, 429.

[7] E. Balikci, A. Deal and R. Abbaschian, J. Crystal Growth, 271 (2004) 37.

[8] O. Patzold, K. Jenkner, S. Scholz and A. Croll, J. Crystal Growth, 277 (2005) 37.

[9] W. Palosz, M. P. Voltz, S. Cobb, S. Motakef, F. R. Szofran, J. Crystal Growth, 277 (2005) 124. 
[10] M. P. Volz, M. Schweizer, B. Raghothamachar, M. Dudley, J. Szoke, S.D. Cobb and F.R.Szofran, J. Crystal Growth, 290 (2006) 446.

[11] T. Duffar, I. Paret-Harter, and P. Dusserre, J. Crystal Growth 100 (1990) 171.

[12] T. Duffar, P. Boiton, P. Dusserre, and J. Abadie, J. Crystal Growth 179 (1997) 397.

[13] W. R. Wilcox, L.L.Regel, Microgravity Sci. Technol. 8 (1995) 56.

[14] C. Stelian, A. Yeckel, and J.J. Derby, Influence of thermal phenomena on crystal reattachment during the dewetted Bridgman growth, J. Crystal Growth, in press.

[15] J. Fainberg, D. Vizman, J. Friedrich, G. Mueller, Journal of Crystal Growth 303 (2007) 124.

[16] T. Duffar, I. Paret-Harter, and P. Dusserre, J. Crystal Growth 100 (1990) 171.

[17] T. Duffar, P. Boiton, P. Dusserre, and J. Abadie, J. Crystal Growth 179 (1997) 397.

[18] L. Braescu, Journal of Colloid and Interface Science 319 (2008) 309.

[19] St. Balint, L. Braescu, L. Sylla, S. Epure, T. Duffar, Journal of Crystal Growth 310 (2008) 1564 .

[20] J.J. Derby and R.A. Brown, J. Crystal Growth 83 (1987) 137.

[21] J.J. Derby, L.J. Atherton, P.D. Thomas, and R.A. Brown, J. Scientific Computing 2 (1987) 297.

[22] L.J. Atherton, J.J. Derby, and R.A. Brown, J. Crystal Growth 84 (1987) 57-78.

[23] J.J. Derby, L.J. Atherton, and P.M. Gresho, J. Crystal Growth 97 (1989) 792.

[24] J.J. Derby and Q. Xiao, J. Crystal Growth 113 (1991) 575.

[25] Q. Xiao and J.J. Derby, J. Crystal Growth 139 (1994) 147.

[26] A. Yeckel, A.G. Salinger, and J.J. Derby, J. Crystal Growth 152 (1995) 51.

[27] A. Pandy, A. Yeckel, M. Reed, C. Szeles, M. Hainke, G. Müller, and J.J. Derby, Journal of Crystal Growth 276 (2005) 133.

[28] A. Yeckel, A. Pandy, and J.J. Derby, International Journal for Numerical Methods in Engineering 67 (2006)1763.

[29] J.J. Derby, L. Lun, and A. Yeckel, J. Crystal Growth 303 (2007) 114.

[30] A. Yeckel, L. Lun, and J.J. Derby, J. Comp. Phys. submitted. 


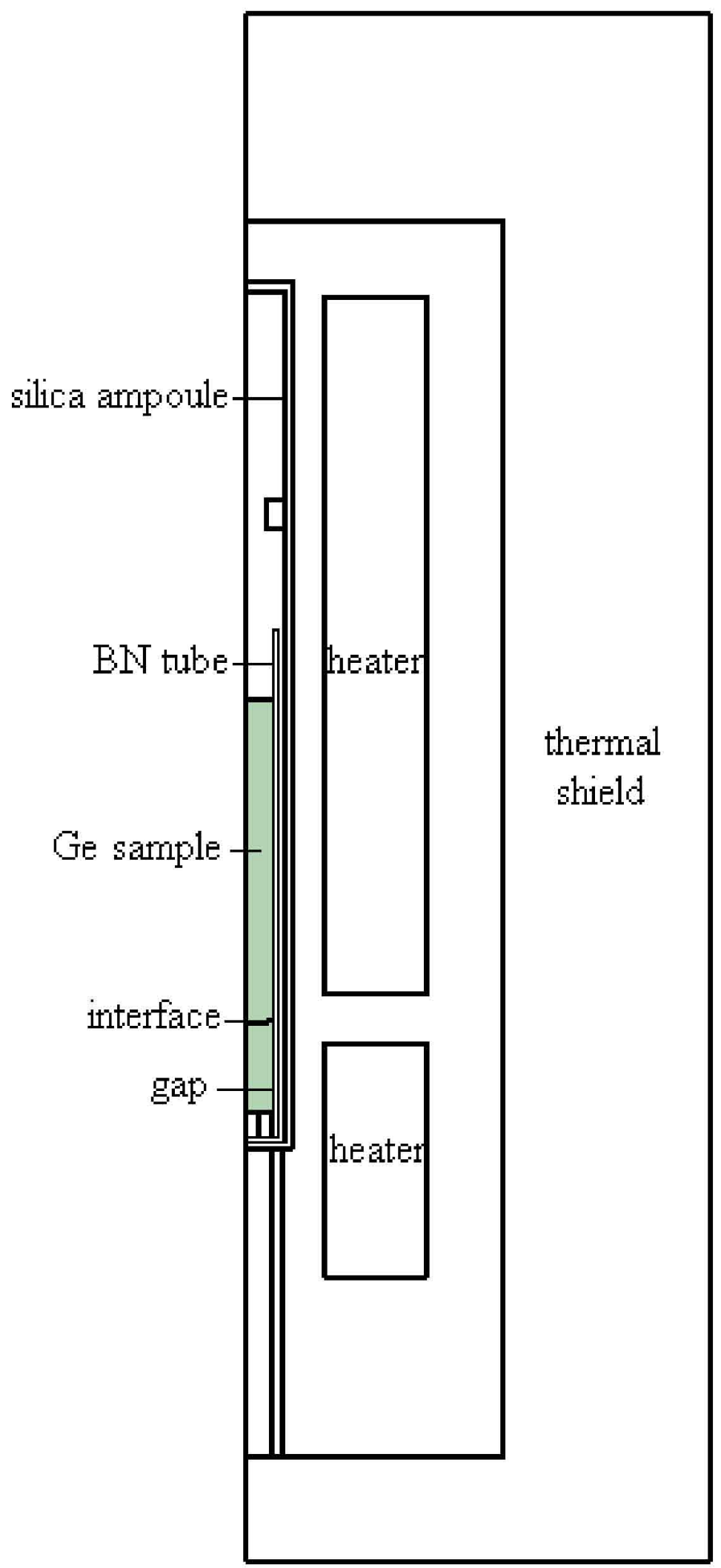

Figure 1: Schematic diagram of the computational domains for the Bridgman configuration with two heaters employed by Palosz et al. [9]. 


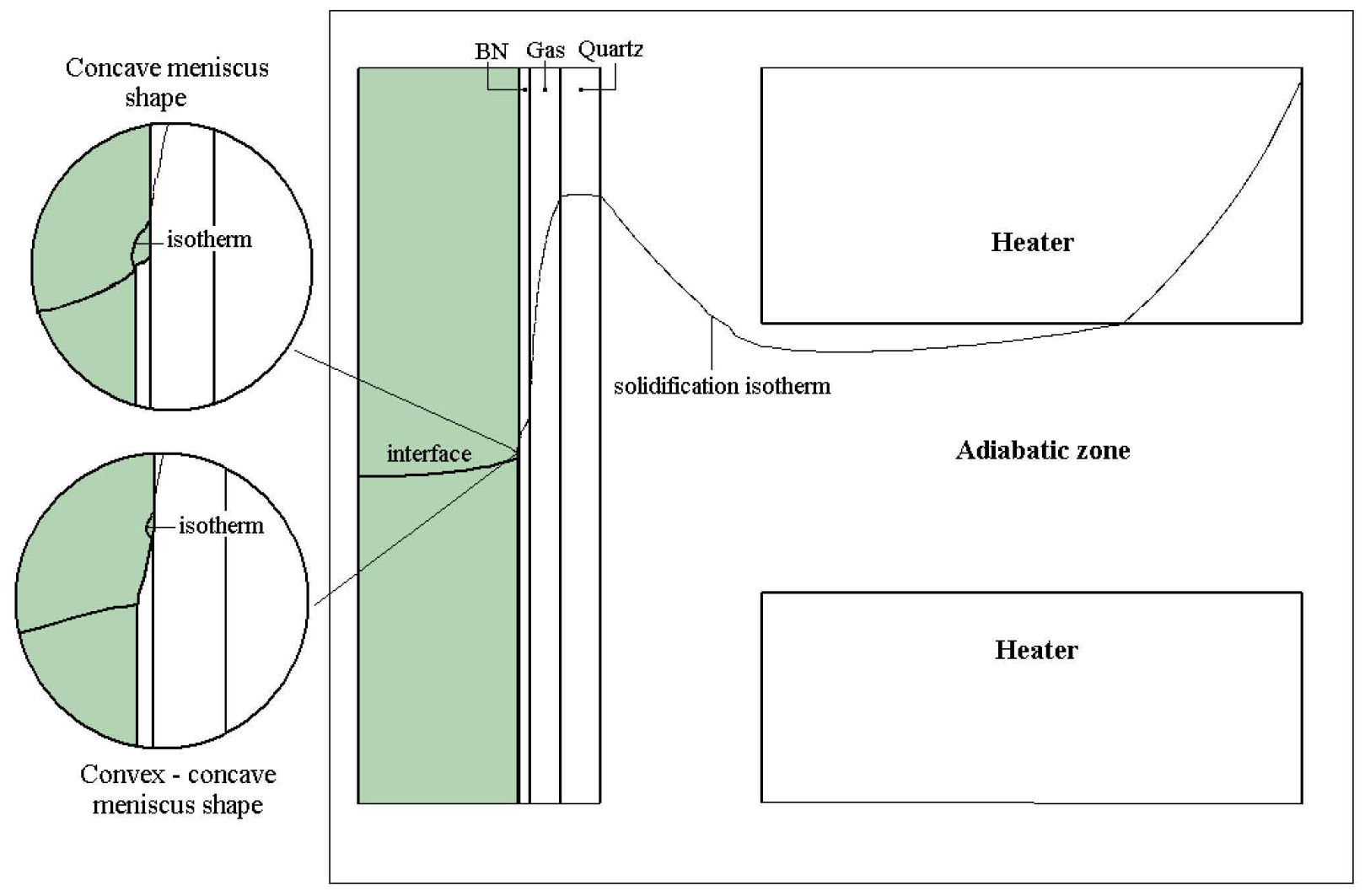

Figure 2: Relative positions of the solidification isotherm and meniscus predicted for the configuration using a $\mathrm{BN}$ inner tube; the position of the solid-liquid interface is located in the center of the adiabatic zone. Two detailed views show cases for a convex-concave meniscus (below) and a concave meniscus (above). 

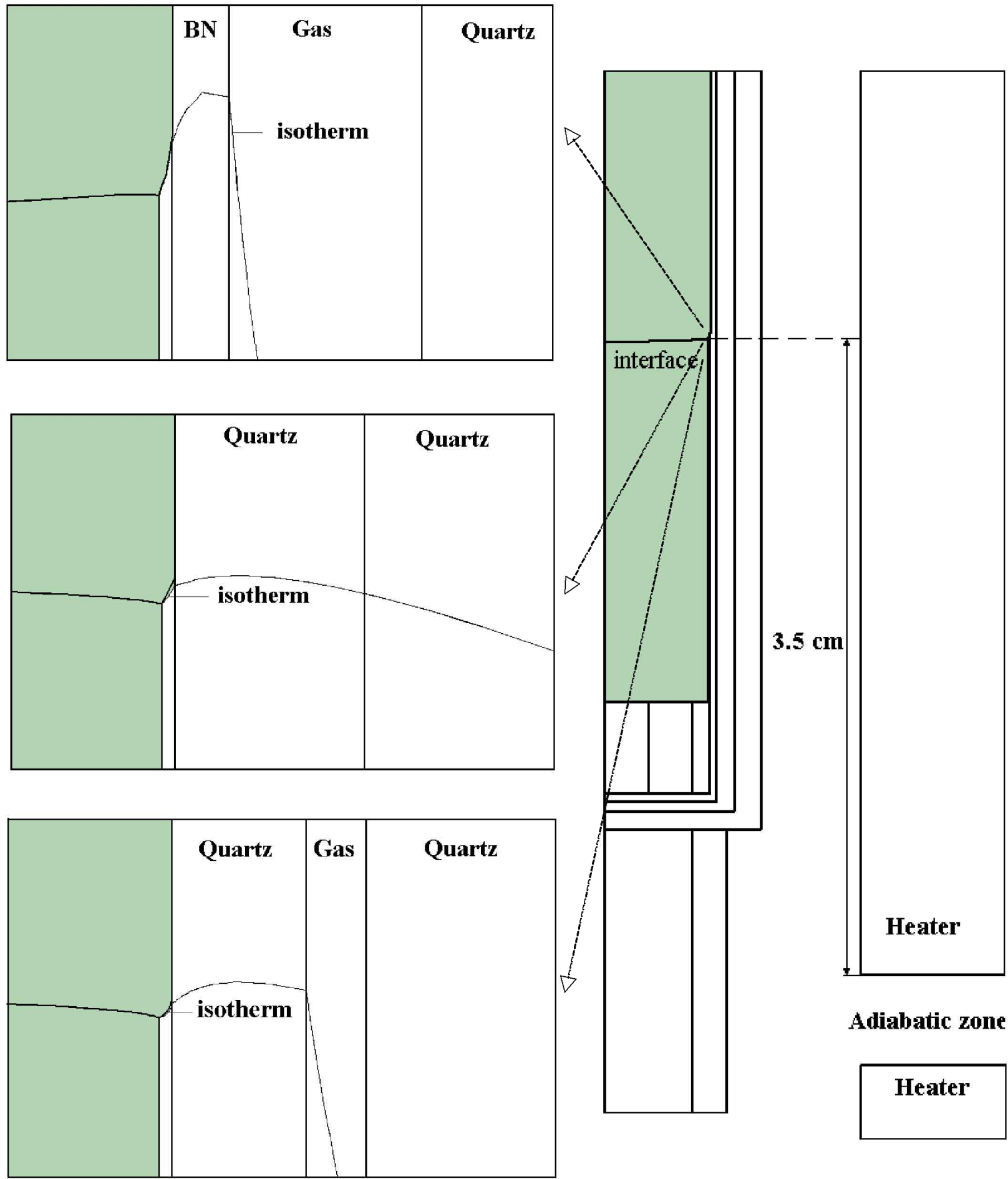

Figure 3: Relative positions of the solidification isotherm and meniscus predicted for several different ampoule-crucible configurations; the interface is positioned at $3.5 \mathrm{~cm}$ above the adiabatic zone. 

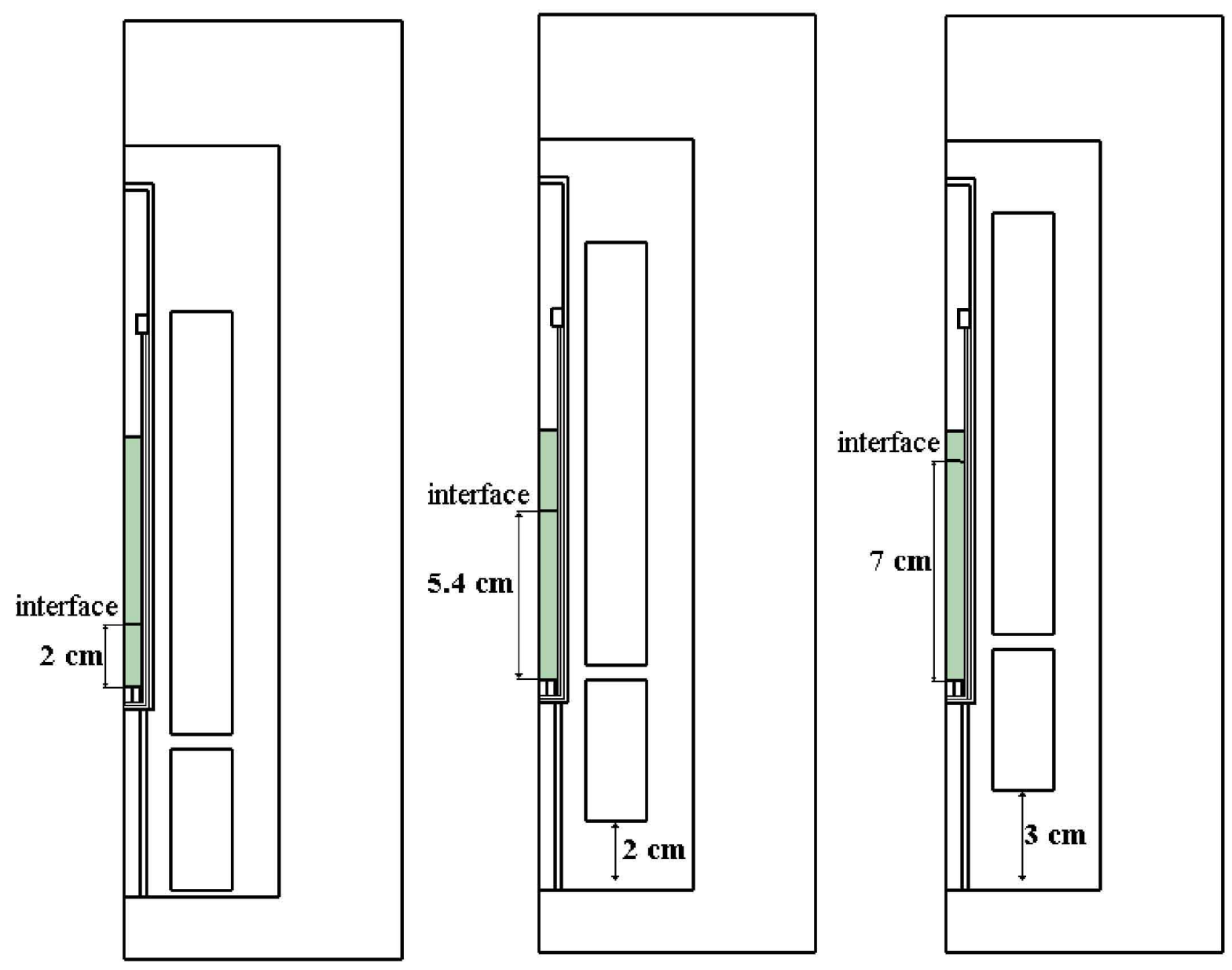

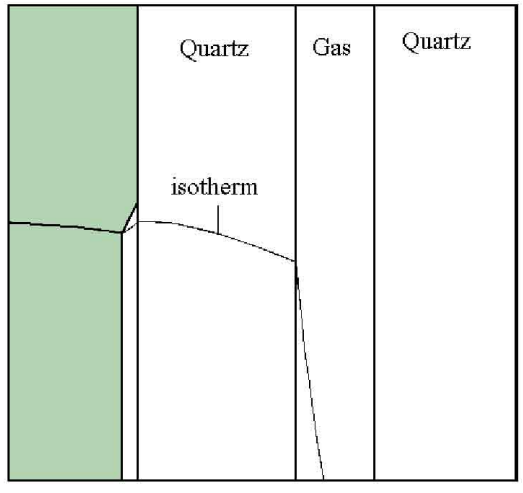

a)

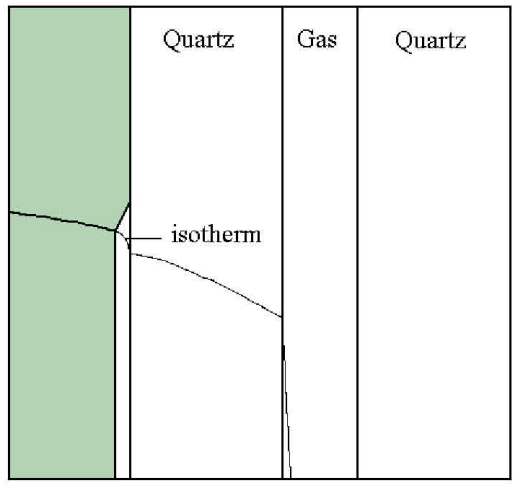

b)

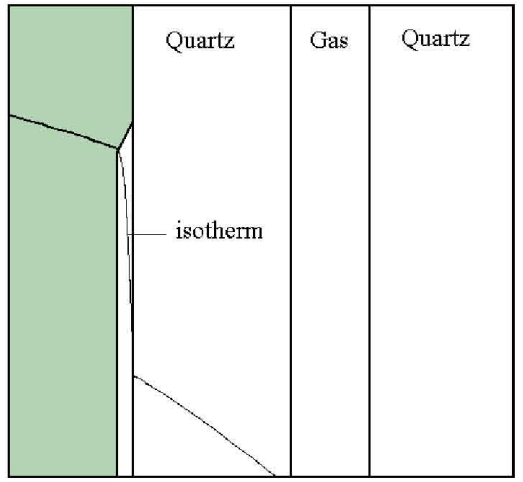

c)

Figure 4: Evolution of the position of the solidification isotherm when the furnace is translated up to simulate growth; the ampoule configuration consists of two quartz tubes separated by gas: (a) initial state; (b) heaters translated by $2 \mathrm{~cm}$; (c) heaters translated by $3 \mathrm{~cm}$. 

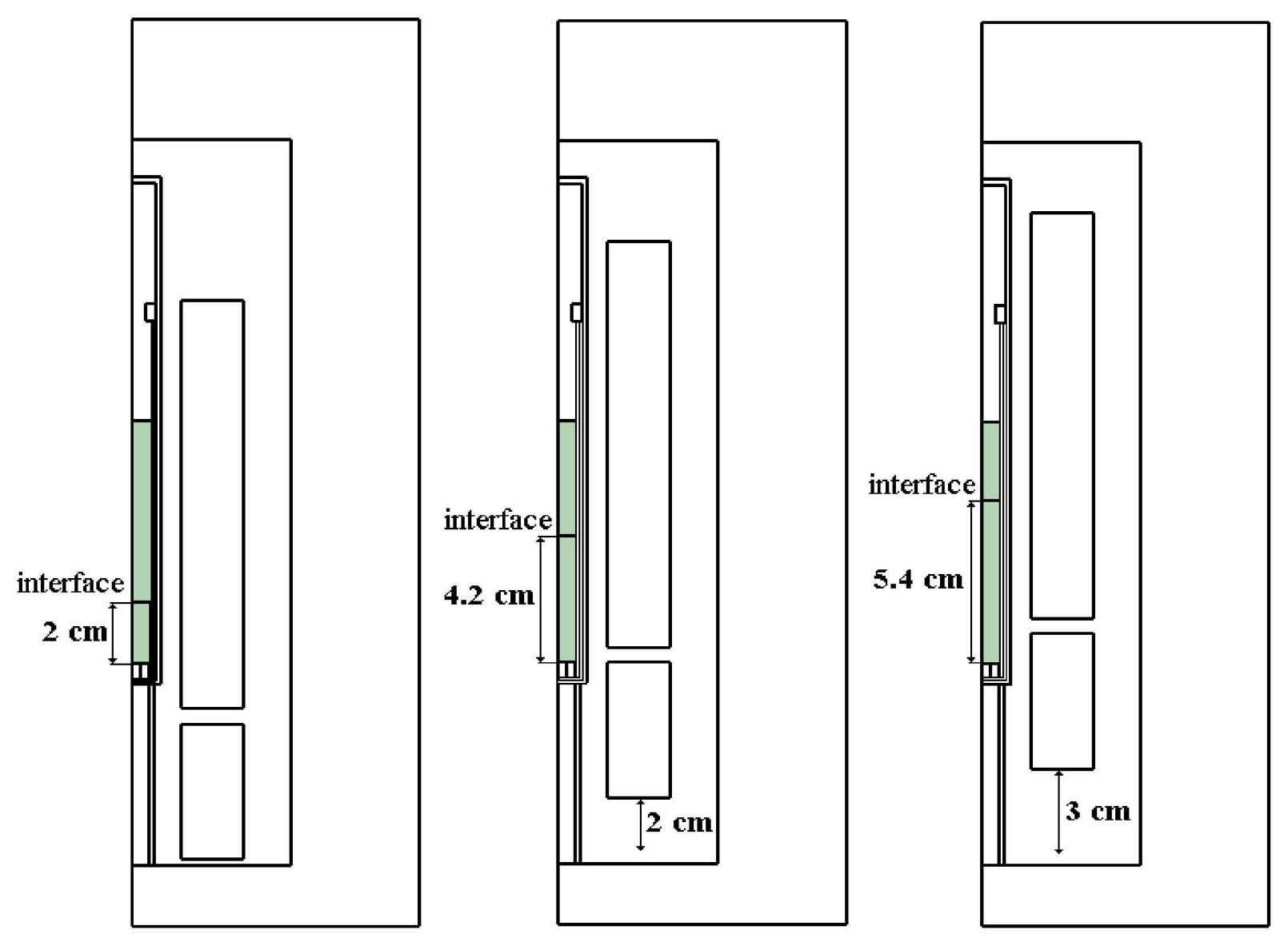

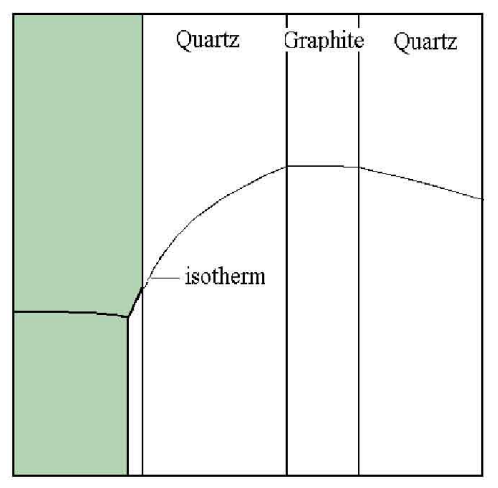

a)

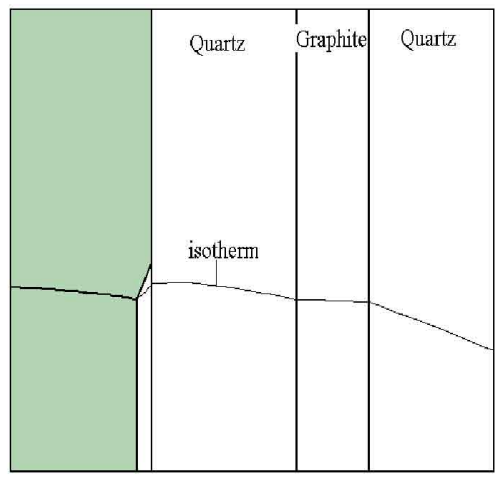

b)

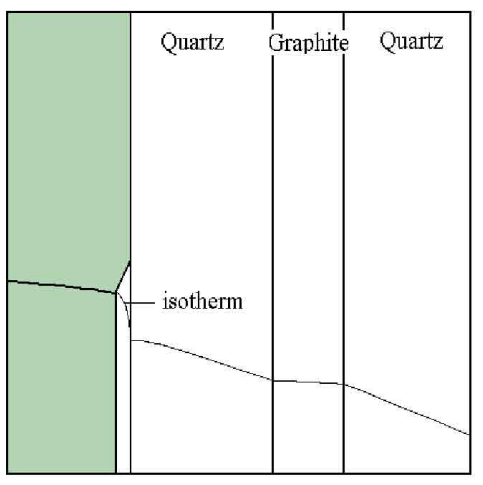

c)

Figure 5: Evolution of the position of the solidification isotherm when the furnace is translated up to simulate growth; the ampoule configuration consists of two quartz tubes with a layer of graphite between: (a) initial state; (b) heaters translated by $2 \mathrm{~cm}$; (c) heaters translated by $3 \mathrm{~cm}$. 


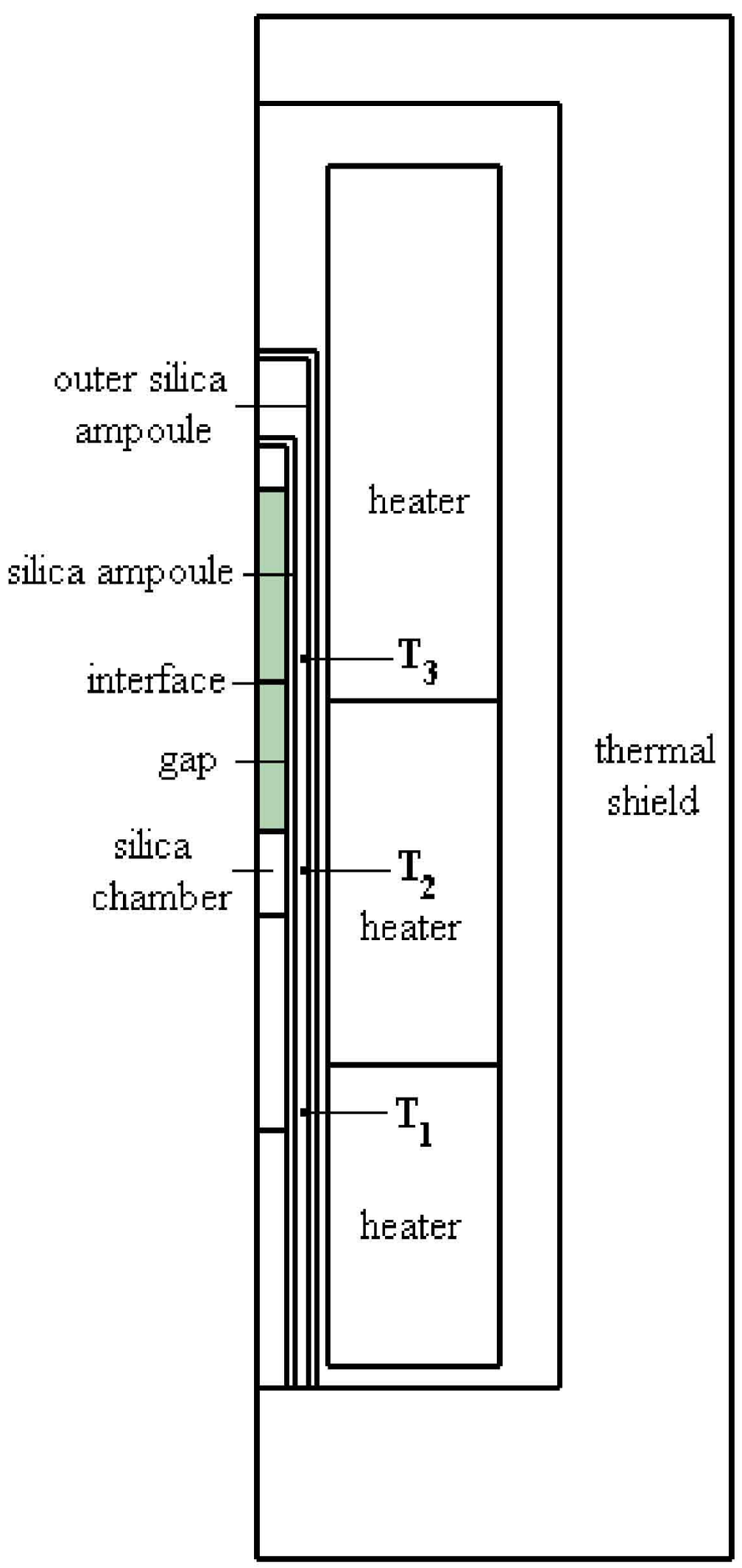

Figure 6: Schematic diagram of the computational domains for the VGF configuration with three heaters employed by Chevalier et al. [5]. 


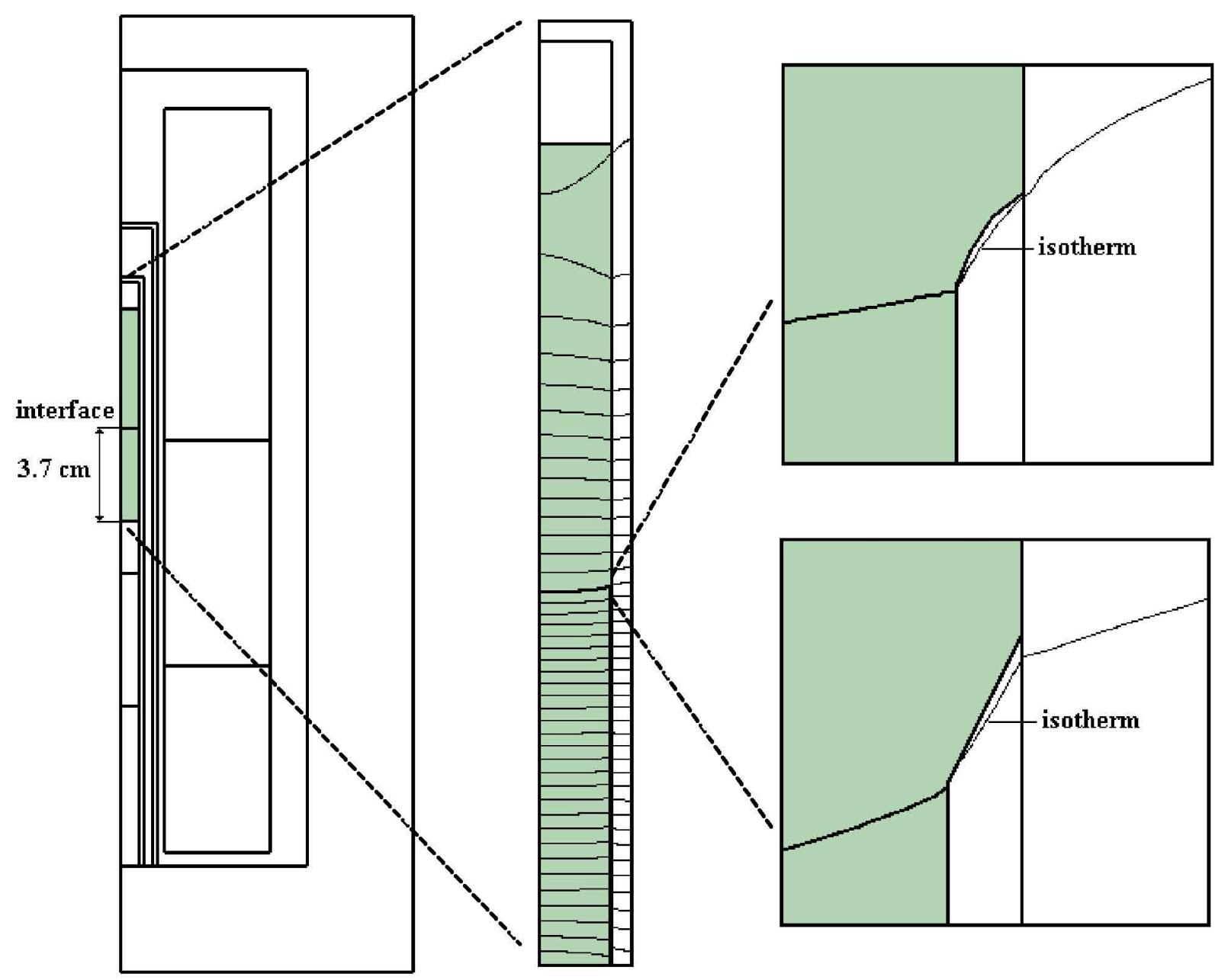

Figure 7: Thermal field predicted for the initial configuration of successful detached growth described in [5]. Two detailed views show relative positions of the solidification isotherm and meniscus for a convex meniscus (above) and a straight-line approximation to the meniscus (below). 


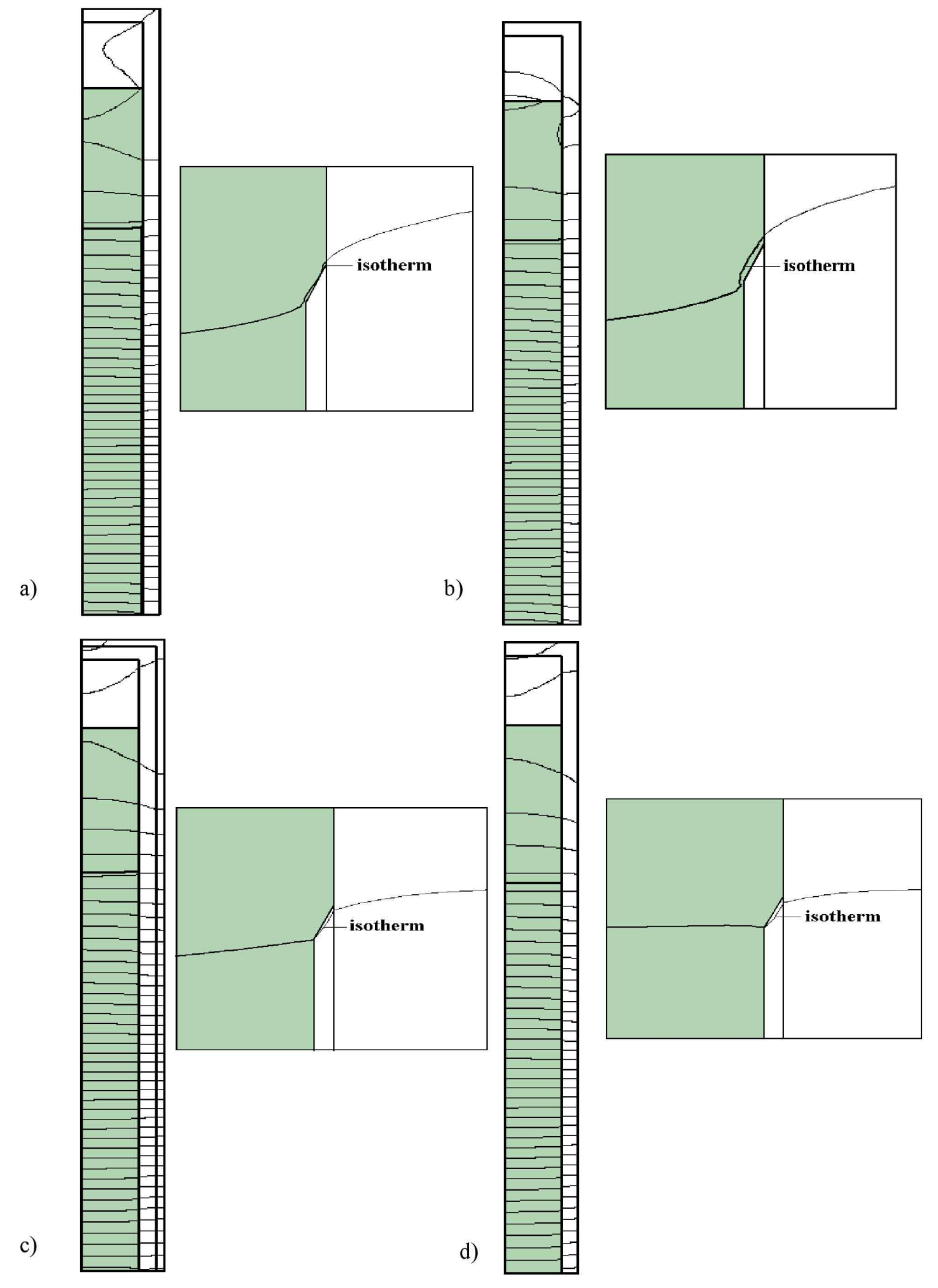

Figure 8: Thermal field predicted for an advanced position of the interface, corresponding to $5.9 \mathrm{~cm}$ length of the solid. Detailed views show relative positions of the solidification isotherm and meniscus. Case for (a) semitransparent quartz ampoule; (b) completely transparent quartz ampoule; (c) semitransparent quartz ampoule with its exterior coated by a graphite layer; (d) opaque quartz ampoule. 

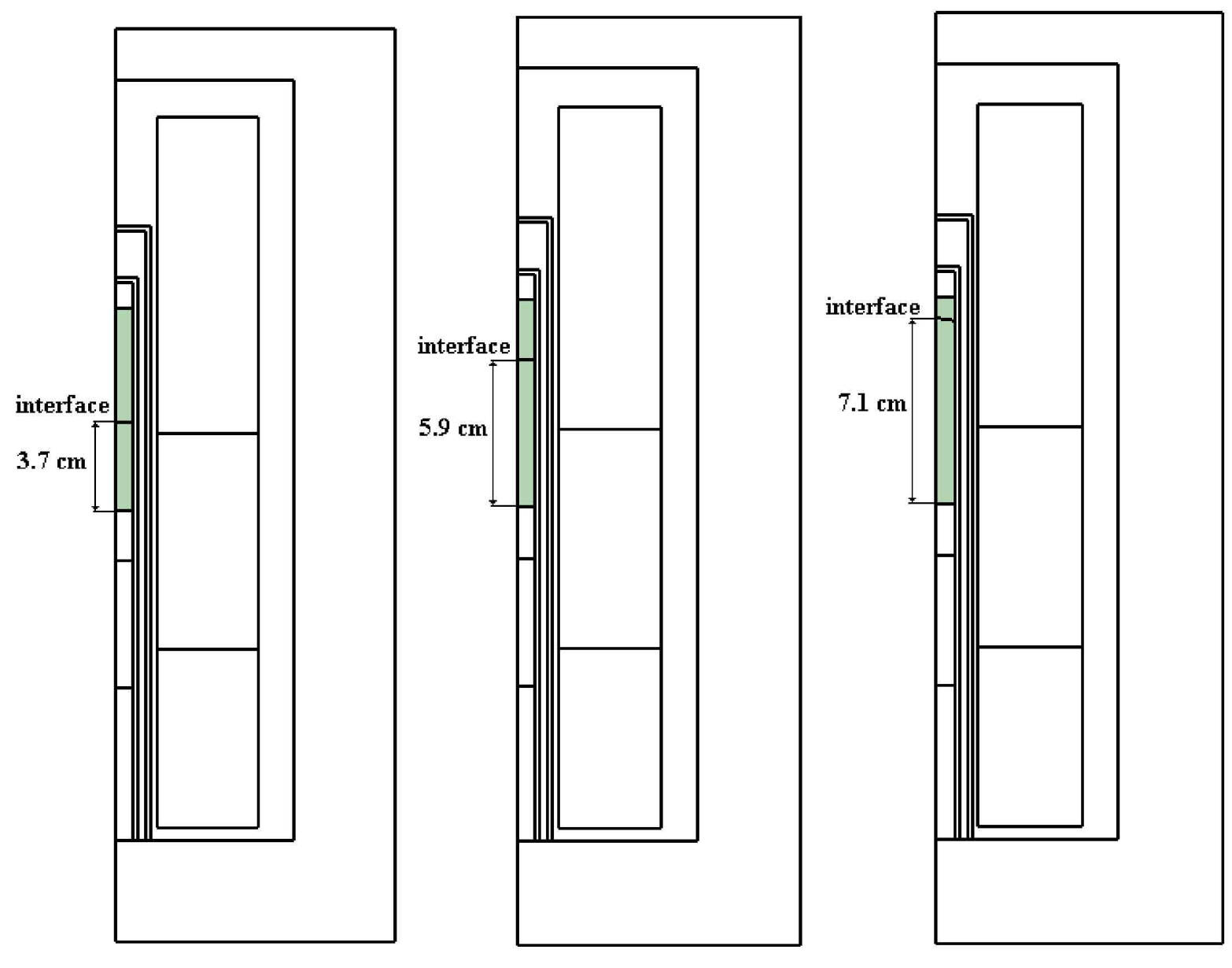

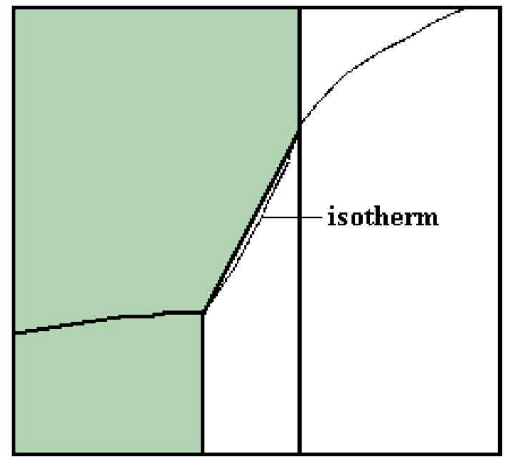

a)

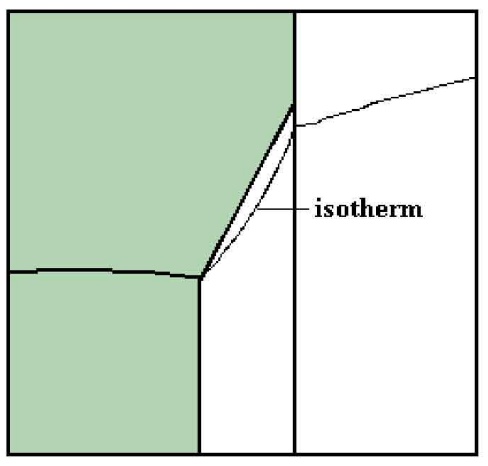

b)

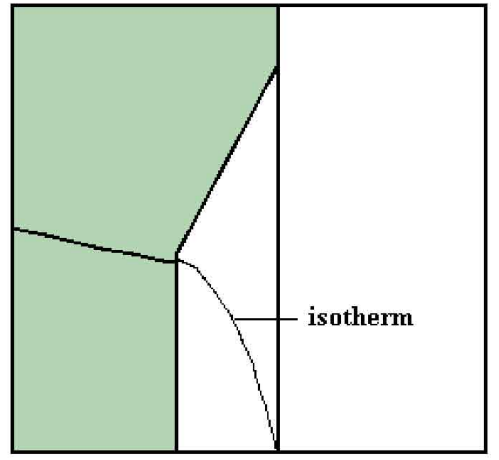

c)

Figure 9: Evolution of the position of the solidification isotherm when the furnace is powered down to simulate growth under the conditions described in [5]: (a) initial state (seed length of $3.7 \mathrm{~cm}$ ); (b) solid length of $5.9 \mathrm{~cm}$; (c) solid length of $7.1 \mathrm{~cm}$. 